\title{
Mismatch repair deficiency occurs very rarely in seminomas
}

\author{
David Dum $^{1 \#}$, Stefan Steurer ${ }^{1 \#}$, Ronald Simon ${ }^{1}$, Pia Victoria Zimmermann ${ }^{1}$, Eike Burandt ${ }^{1}$, \\ Till Sebastian Clauditz ${ }^{1}$, Margit Fisch' ${ }^{2}$, Michael Rink ${ }^{2}$, Roland Dahlem², Wolfgang Höppner ${ }^{3}$, \\ Henrik Zecha ${ }^{4}$, Ousman Doh ${ }^{5}$, Cord Matthies ${ }^{6}$, Waldemar Wilczak ${ }^{1}$, Guido Sauter ${ }^{1}$, Christoph Fraune ${ }^{1}$ \\ ${ }^{1}$ Institute of Pathology, University Medical Center Hamburg-Eppendorf, Hamburg, Germany; ${ }^{2}$ Department of Urology, University Medical \\ Center Hamburg-Eppendorf, Hamburg, Germany; ${ }^{3}$ Department of Urology, Itzehoe Medical Center, Itzehoe, Germany; ${ }^{4}$ Department of Urology, \\ Albertinen Clinic, Hamburg, Germany; ${ }^{5}$ Department of Urology, Regio Medical Center Elmshorn, Elmshorn, Germany; ${ }^{6}$ Department of Urology, \\ Bundeswehr Hospital Hamburg, Hamburg, Germany \\ Contributions: (I) Conception and design: D Dum, S Steurer, R Simon, M Fisch, G Sauter, C Fraune, M Fisch, M Rink, R Dahlem, W Höppner, H \\ Zecha, O Doh, C Matthies; (II) Administrative support: W Wilczak, R Simon, TS Clauditz, E Burandt; (III) Provision of study materials or patients: \\ M Fisch, M Rink, R Dahlem, W Höppner, H Zecha, O Doh, C Matthies; (IV) Collection and assembly of data: D Dum, S Steurer, R Simon, PV \\ Zimmermann, E Burandt, TS Clauditz, W Wilczak, G Sauter, C Fraune; (V) Data analysis and interpretation: E Burandt, TS Clauditz, W Wilczak, \\ G Sauter; (VI) Manuscript writing: All authors; (VII) Final approval of manuscript: All authors. \\ \#These authors contributed equaly to this work. \\ Correspondence to: Prof. Dr. Ronald Simon. Institute of Pathology, University Medical Center Hamburg-Eppendorf, Martinistr. 52, 20246 Hamburg, \\ Germany. Email R.Simon@uke.de.
}

Background: Dense tumor-associated lymphocyte infiltration is linked to mismatch repair (MMR) deficiency in colorectal and endometrial cancer. MMR deficiency is of high clinical importance as MMR deficient cancers tend to react favorably to treatment with immune checkpoint inhibitors. Strong lymphocytic infiltration is a morphological hallmark of seminomas. We thus asked whether seminomas may exhibit MMR deficiency at relevant frequency.

Methods: To screen for tumors with MMR deficiency, protein expression of MLH1, PMS2, MSH2, and MSH6 was analyzed by immunohistochemistry (IHC) on a tissue microarray (TMA) containing 574 seminomas.

Results: In total, 536 cases were evaluable resulting in 481 seminomas with unequivocally intact MMR protein expression. In 55 cancers, one or several IHC stains were equivocal and lacked detectable MMR protein in both tumor and stromal cells. Large section IHC analysis of all 55 equivocal cases demonstrated substantial staining issues due to improper fixation in 54 cases and identified one tumor with clear-cut MLH1 and PMS2 protein loss. This seminoma showed homogeneous loss of MLH1 and PMS2 in the entire tumor mass whereas minor adjacent foci of associated germ cell neoplasia in situ (GCNIS) were MMR intact. Polymerase chain reaction (PCR) analysis using the 5 microsatellite loci of the "Bethesda Panel" revealed instability in 1 of 4 interpretable loci ("MSI-low") and additional instability of the complex tetra-penta repeat locus MYCL1 in this tumor.

Conclusions: In summary, one single seminoma with MMR deficiency, characterized by protein loss of MLH1 and PMS2, was identified among 536 interpretable seminomas (0.19\%). MMR deficiency is not a relevant determinant of lymphocyte influx in seminoma.

Keywords: Seminoma; mismatch repair (MMR); immunohistochemistry; microsatellite instability (MSI)

Submitted Oct 20, 2020. Accepted for publication Dec 15, 2020.

doi: $10.21037 /$ tau-20-1355

View this article at: http://dx.doi.org/10.21037/tau-20-1355 


\section{Introduction}

Microsatellite instability (MSI) is of considerable topical interest because-irrespective of their site of origincancers exhibiting MSI are likely to respond favorably to immune checkpoint inhibitor therapy $(1,2)$. MSI reflects a hypermutator phenotype inducing a high mutational load in affected tumors, typically due to a deficient mismatch repair (MMR) system unable to resolve short slippage DNA errors that occur during cell cycle. MSI can be detected directly with polymerase chain reaction (PCR)-based methods or indirectly by identifying loss of expression of the MMR proteins MLH1, PMS2, MSH2, and MSH6 by immunohistochemistry (IHC) (3).

MSI occurs in various malignancies $(4,5)$. Highest frequencies have been reported for endometrial (7-36\%) $(6,7)$, colorectal $(5-23 \%)(8-10)$, and stomach cancer $(6-28 \%)(11-15)$, but various recent studies have demonstrated that MSI can be found in virtually all cancer types at a frequency of around 1\% $(4,5)$. On a morphological level, neoplasms with MSI are often characterized by a markedly increased density of tumorassociated lymphocytes (16-18). For seminoma-the most common type of testicular germ cell tumors-a strong tumor-associated lymphocytic reaction is so common that it represents an important morphologic hallmark for establishing this diagnosis (19). In a recent comparative analysis of the density of CD8 positive lymphocytes across 84 different tumor types, seminoma ranked 7th with 424 CD8 positive lymphocytes per $\mathrm{mm}^{2}$ (20). The role of MSI in seminoma is unclear, however. Reported rates of MSI detected directly by next generation sequencing (NGS) or PCR range from 0-27\% (21-27). Only two studies have used currently accepted criteria for immunohistochemical determination of MMR deficiency and did not find cases with MMR protein loss among 74 and 77 seminomas $(28,29)$.

In order to clarify the prevalence of MSI in seminomas and to assess its possible heterogeneity, a tissue microarray (TMA) containing 574 seminomas was constructed. The analyses included a TMA-based IHC evaluation of the protein expression of the MMR genes MLH1, PMS2, MSH2, and MSH6 as well as a subsequent validation on large sections by IHC and PCR. We present the following article in accordance with the MDAR reporting checklist (available at: http://dx.doi.org/10.21037/tau-20-1355).

\section{Methods}

\section{Subjects}

A TMA comprising samples from 574 seminomas and various control tissues was constructed from archived tumor material from orchiectomy specimens of patients diagnosed with seminoma at the University Medical Center HamburgEppendorf between 2009 and 2018 using a $0.6 \mathrm{~mm}$ tumor containing tissue core per case. TMA construction was described earlier (30). The study was conducted in accordance with the Declaration of Helsinki (as revised in 2013). Utilization of archived remnants of diagnostic tissues for manufacturing of TMAs and their analysis for research purposes as well as patient data analysis has been approved by local laws (HmbKHG, \$12) and by the local ethics committee (Ethics commission Hamburg, WF-049/09). According to local laws, informed consent was not required for this study.

\section{Main reagents and instruments}

Immunohistochemical analyses. Freshly taken TMA sections were immunostained on one day using an automated immunostainer (Dako/Agilent Autostainer Link 48). Primary antibody specific for MLH1 (clone ES05, mouse), PMS2 (clone EP51, rabbit), MSH2 (clone FE11, mouse), and MSH6 (clone EP49, rabbit) (all Ready-to-Use, all from DAKO, Glostrup, Denmark) was applied for 20 minutes (MLH1, MSH2, MSH6) or 30 minutes (PMS2). Bound antibody was visualized using the EnVision Kit (Dako, Glostrup, Denmark) according to the manufacturer's directions. If no unequivocal nuclear staining in tumor cells was observed, staining was recorded as negative (0). Clear-cut nuclear staining in tumor cells was scored as mild (1+), moderate (2+), or strong (3+) depending on the average staining intensity of all tumor cells with positive nuclear staining. The percentage of positive tumor cells was recorded estimating the fraction of tumor cells with positive nuclear staining $(1+, 2+$, or $3+)$ from all analyzable tumor cells on the respective TMA spot. In spots showing a negative $(0)$ result in the tumor cells, presence $(+)$ or absence (-) of nuclear staining in peritumoral stromal or inflammatory cells was additionally recorded as an internal control. For TMA spots with suspected MMR deficiency or with an equivocal staining result, IHC was repeated on a large section of the routinely archived tumor material. In 
case of confirmed MMR deficiency, all available archived tumor-containing blocks were also analyzed by IHC.

\section{PCR analysis}

One seminoma with clear-cut MMR deficiency was identified by TMA screening and subsequently analyzed for MSI. A PCR-based assay was performed (MSI Analysis System; Promega, Madison, WI), incorporating the five microsatellite loci of the "Bethesda Panel", which include two mononucleotide repeats (BAT25, BAT26) and three dinucleotides repeats (D2S123, D5S346 and D17S250). One complex tetra-penta repeat locus (MYCL1) was also analyzed. DNA from tumor and non-neoplastic tissue was extracted from a large section of the respective tumor block corresponding to the TMA spot. MSI-high was recorded when at least $2(\geq 40 \%)$ of the Bethesda markers showed instability (i.e., length variation compared to control tissue) and MSI-low if any one of the analyzed loci showed instability, otherwise microsatellite stability (MSS) was assumed.

\section{Statistical analysis}

No statistical calculations were performed in this study.

\section{Results}

\section{TMA screening and large section validation}

A total of 38 of 574 arrayed seminoma samples completely lacked tissue or tumor on at least one of the immunostained TMA slides and were thus excluded from the analysis. Among the remaining 536 seminomas, none showed clear-cut MMR protein loss. In 481 tumors all 4 MMR proteins were unequivocally retained. In the remaining 55 tumors, at least one MMR protein could not be evaluated on the respective TMA spot because a lack of staining was observed in both tumor and adjacent stromal and inflammatory cells. To exclude that any MMR deficiency was not identified among the tumors with inconclusive results ( $\mathrm{n}=55$ ), IHC for the respective MMR protein(s) was repeated on corresponding large sections $(n=76)$. Large section IHC typically showed a significant gradient of immunoreactivity with clear-cut staining of tumor and nontumorous cell nuclei in the periphery of the tumor whereas central tumor areas, which were typically represented on the TMA, were not or only poorly stained (Figure 1). However, in one seminoma unambiguous MMR deficiency was identified, characterized by protein loss of MLH1 and PMS2 in tumor cells while adjacent non-neoplastic cells were strongly positive (Figure 2). MSH2 and MSH6 were both retained in the tumor cells. Overall, one of 536 interpretable seminomas was found MMR deficient (0.19\%).

\section{MSI validation}

PCR analysis of the MMR deficient tumor revealed the following results: BAT25: stable, D12S250: stable, BAT26: instable, D5S346: stable, D2S123: not interpretable. Accordingly, the tumor was classified as MSI-low. In addition, MCYL1, a complex penta-tetra-repeat not part of the Bethesda Panel was also found instable.

\section{Heterogeneity analysis of MMR deficiency}

Thorough IHC analysis of all 3 tumor containing blocks of the seminoma with MSI revealed uniform MMR protein loss of MLH1 and PMS2 throughout the entire tumor. Minor adjacent foci of associated germ cell neoplasia in situ (GCNIS) that were visible on one tissue slide were MMR intact.

\section{Discussion}

The successful analysis of 536 seminomas in this study lead to the identification of only one tumor with unequivocal MMR deficiency. The fact that the entire tumor mass exhibited a distinct loss of MLH1 and PMS2 while these proteins were still expressed in GCNIS shows that MMR deficiency must have occurred early in the development of this neoplasia, probably around the transition from non-invasive to invasive seminoma. It is of interest that only one of 4 interpretable markers of the "Bethesda Panel" was instable in this seminoma resulting in a formal categorization as MSI-low. Biologically relevant MSI in this tumor is, however, supported by instability of MCYL1, a complex penta-tetra-repeat not belonging to the "Bethesda Panel". The "Bethesda Panel", a selection of 5 specific mono- and dinucleotide repeats, has been developed based on data derived from colorectal cancers (31). As the likelihood for the occurrence of instability in individual repeat loci may depend on the transcriptional activity of a genomic region, individual microsatellite loci may not be equally affected by frameshifts in all tissues and cell types $(32,33)$. The Bethesda Panel may thus not be equally suited for MSI detection across different tumor types. 


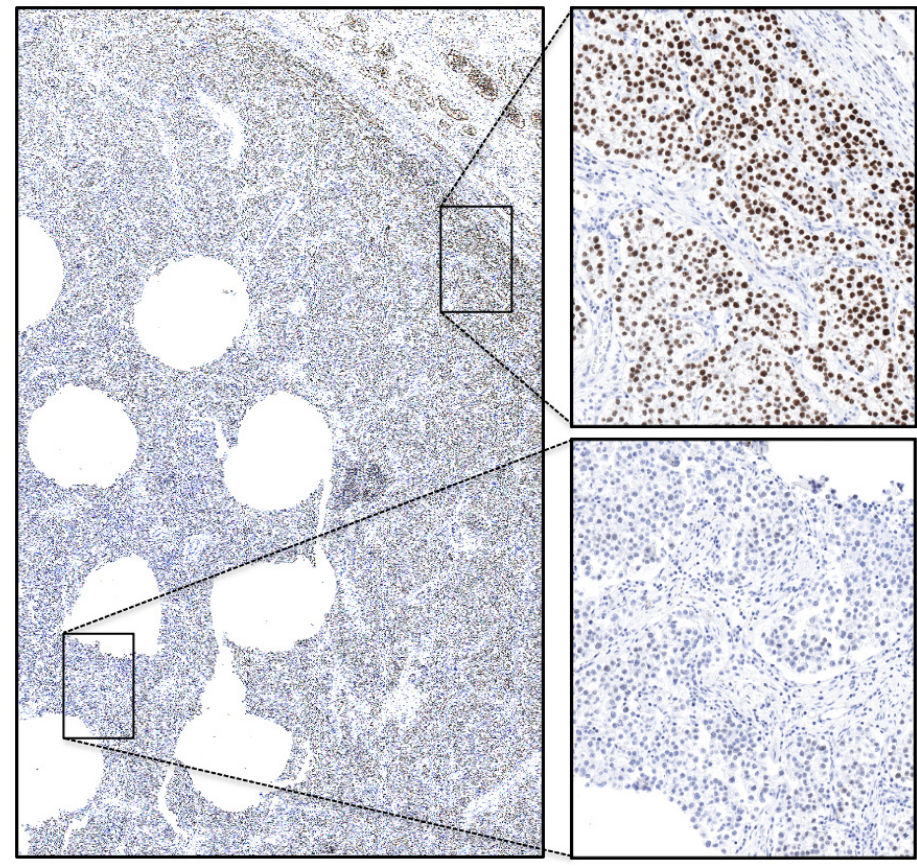

Figure 1 Large section IHC of a tumor with inconclusive TMA screening results revealed pronounced staining variability due to suboptimal fixation, here shown for MSH6. Strong staining was observed in the periphery of the tumor with gradual decrease of staining intensity towards central areas adjacent to punch holes from TMA construction. The corresponding TMA spot (not shown) taken from the central area was inconclusive due to negative staining both in tumor and control cells. Original magnifications $1.6 \times$ and $40 \times$. IHC, immunohistochemistry; TMA, tissue microarray.

Our data suggest that seminoma is a neoplasia with a particularly low rate of MSI/MMR deficiency $(0,19 \%)$, indicating that the prominent inflammatory infiltrate typically present in seminomas is unrelated to the MMR status. This notion largely fits with the results of several earlier studies. A previous report using standardized PCRbased methods (incorporating a modified "Bethesda Panel") to detect MSI has not identified any MSI-high cases among 32 seminomas (21). Necchi et al. also failed to find MSI-high tumors within 23 relapsed seminomas (27). Recent studies employing NGS to analyze MSI in 150 (5) and 368 (4) germ cell tumors identified only 1 MSI case in total, but it was not specified whether this case was a seminoma or not. The reason for the rarity of MSI in seminoma is unclear. It might be speculated that the rate of seminomas with MSI is higher in reality but that most of the highly immunogenic MSI seminomas are eliminated by their inflammatory microenvironment. It is indeed a hallmark of seminomas that an estimated $10 \%$ of these tumors spontaneously regress to residual scar formations (i.e., "burned-out seminoma") (34). Of note, studies suggesting higher rates of MSI in seminoma do exist. Early PCR-based investigations did, however, use less stringent criteria and/ or largely expanded panels of microsatellite loci to define MSI (23,25,35). For instance, Velasco et al. found MSI in 11 of $44(25 \%)$ seminomas using a 10 -marker panel and a comparatively low threshold of $30 \%$ to define MSI (25). A recent work by Lobo et al. supports our observation that the inflammatory infiltrate in seminoma is unrelated to MMR status (36). By means of a thorough characterization of the immune cell infiltrate in 271 tumor samples of testicular germ cell tumors, the authors demonstrated that both CD20- and CD3-positive immune cells were not associated with the expression levels of any of the four MMR proteins MLH1, PMS2, MSH2, and MSH6 in the subset of seminomas (36).

Most previous IHC based studies on MMR expression in germ cell tumors used a quantitative approach to correlate the relative expression (low versus high) with other tumor parameters as well as clinical outcome rather than applying stringent criteria for defining loss of MMR protein expression $(22,24,25)$. Two studies analyzing MLH1, PMS2, 

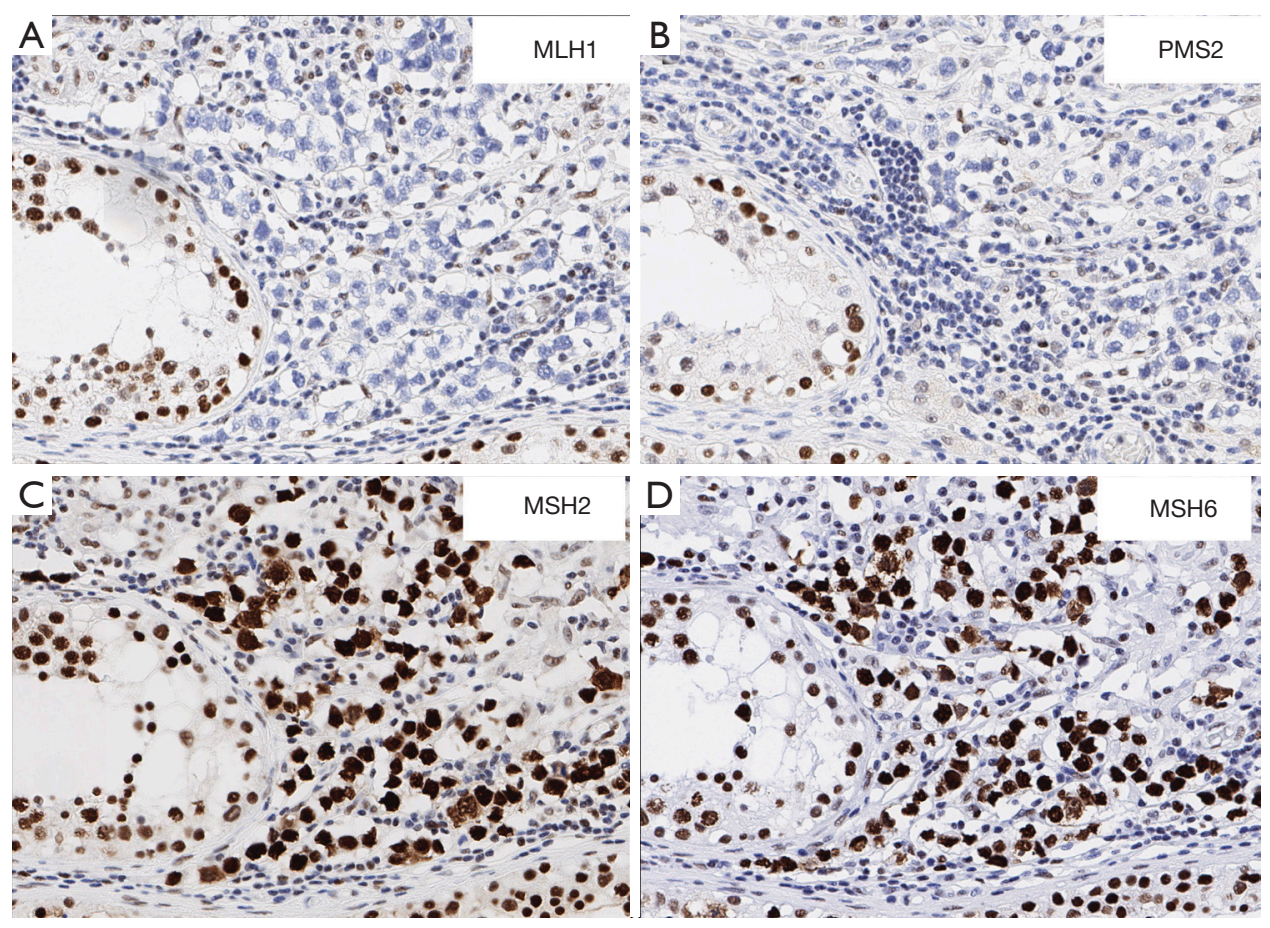

Figure 2 One seminoma with MMR deficiency and MSI confirmed by PCR. IHC on large sections revealed protein loss for MLH1 (A) and PMS2 (B) in tumor cells, whereas adjacent stromal and inflammatory cells as well as cells of the residual seminiferous tubules were positive. MSH2 (C) and MSH6 (D) were retained in the tumor cells. Original magnifications 40×. MMR, mismatch repair; MSI, microsatellite instability; PCR, polymerase chain reaction; IHC, immunohistochemistry.

MSH2, and MSH6 by IHC on 20 and 51 seminomas used similar interpretation criteria as in our study and did not find any tumors with MMR protein loss (28,29). However, Olasz et al. used an analogous IHC approach for MLH1, PMS2, MSH2, and MSH6 evaluation as in our study and reported MMR protein loss in 14 of 51 germ cell tumors (37). That none of these tumors with MMR protein loss was MSI-high by PCR analysis may argue for technical issues having contributed to these findings.

Lobo et al. have recently expanded the wide spectrum of Lynch syndrome-associated neoplasms, i.e., hereditary MMR deficient tumors, by reporting a single case of a MMR deficient seminoma due to a MSH2 germline mutation (38). Regarding the seminoma with MLH1 and PMS2 protein loss observed in our study, we cannot reliably distinguish between a sporadic or hereditary cause for MMR deficiency as germline testing was not performed. However, indirect evidence supports a sporadic origin for this tumor. GCNIS adjacent to the seminoma exhibited intact MLH1 and PMS2 protein expression, indicating that MMR loss has occurred during tumor progression. For a hereditary cause, one may expect that MMR loss rather represents an early step of tumor development, at least based on available data derived from Lynch syndrome-associated colorectal carcinomas, where MMR deficiency is already evident in precursor lesions such as very early tubular adenomas and may even be observed in morphologically non-neoplastic crypts $(39,40)$. Moreover, no clinical evidence for Lynch syndrome was reported clinically in our case, further arguing against a hereditary tumor.

Technical IHC issues were indeed considerable in our TMA screening for MMR expression loss. In 55 of 536 analyzable cancers, IHC demonstrated a complete lack of staining in tumor cells for at least one MMR protein. However, MMR protein also lacked detectable expression in adjacent stromal cells, indicating potential staining issues in these cases, which could be confirmed in 54 of 55 tumors in a subsequent analysis using corresponding large sections. In most of these cases, a marked staining gradient was seen with strong staining in the periphery and weak or absent staining in the center of the testis. This can be explained by zonal differences in the quality and extent of formalin fixation, which appears to be particularly common in the testis probably because the dense capsula albuginea hinders 
formalin penetration. In earlier TMA studies using identical IHC protocols for MMR protein evaluation in bladder, prostate, and ovarian cancer, we observed a lower rate of tumors demonstrating TMA-screening based MMR protein loss that could not be confirmed on large sections compared to the present study on testicular cancer (41-43).

It is of note that false negative IHC for MMR proteins may also represent a relevant issue in routine pathology. In a recent study by Cohen et al. observing primary resistance against immune checkpoint inhibitors in 5 out of 38 patients $(13 \%)$ with metastatic colorectal cancer with assumed MSI or MMR deficiency, misdiagnosis of MSI or MMR deficiency was identified as the major cause for treatment failure (44). Given the potential detrimental effects of overtreatment or a mistakenly withheld treatment, the simultaneous use of both IHC and PCR to determine the eligibility for immune checkpoint inhibitors is recommended by a recent consensus of the European Society for Medical Oncology (45).

In summary, our data show that MMR deficiency/MSI occurs exceedingly rare in seminomas but represents an early alteration in affected cases. Thus, MSI is not a cause for the pathognomonic high fraction of tumor infiltrating lymphocytes in this tumor. Fixation variability may represent a particularly relevant problem for IHC analyses of testicular cancers.

\section{Acknowledgments}

We are grateful to Melanie Witt, Inge Brandt, Maren Eisenberg, and Sünje Seekamp for excellent technical assistance.

Funding: None.

\section{Footnote}

Reporting Checklist: The authors have completed the MDAR reporting checklist. Available at http://dx.doi.org/10.21037/ tau-20-1355

Peer Review File: Available at http://dx.doi.org/10.21037/ tau-20-1355

Conflicts of Interest: All authors have completed the ICMJE uniform disclosure form (available at http://dx.doi. org/10.21037/tau-20-1355). All authors have no conflicts of interest to declare.
Ethical Statement: The authors are accountable for all aspects of the work in ensuring that questions related to the accuracy or integrity of any part of the work are appropriately investigated and resolved. The study was conducted in accordance with the Declaration of Helsinki (as revised in 2013). Utilization of archived remnants of diagnostic tissues for manufacturing of TMAs and their analysis for research purposes as well as patient data analysis has been approved by local laws (HmbKHG, \$12) and by the local ethics committee (Ethics commission Hamburg, WF-049/09). According to local laws, informed consent was not required for this study.

Open Access Statement: This is an Open Access article distributed in accordance with the Creative Commons Attribution-NonCommercial-NoDerivs 4.0 International License (CC BY-NC-ND 4.0), which permits the noncommercial replication and distribution of the article with the strict proviso that no changes or edits are made and the original work is properly cited (including links to both the formal publication through the relevant DOI and the license). See: https://creativecommons.org/licenses/by-nc-nd/4.0/.

\section{References}

1. Le DT, Durham JN, Smith KN, et al. Mismatch repair deficiency predicts response of solid tumors to PD-1 blockade. Science 2017;357:409-13.

2. Le DT, Uram JN, Wang H, et al. PD-1 Blockade in Tumors with Mismatch-Repair Deficiency. N Engl J Med 2015;372:2509-20.

3. Baretti M, Le DT. DNA mismatch repair in cancer. Pharmacol Ther 2018;189:45-62.

4. Latham A, Srinivasan P, Kemel Y, et al. Microsatellite Instability Is Associated With the Presence of Lynch Syndrome Pan-Cancer. J Clin Oncol 2019;37:286-95.

5. Bonneville R, Krook MA, Kautto EA, et al. Landscape of Microsatellite Instability Across 39 Cancer Types. JCO Precis Oncol 2017;2017:PO.17.00073.

6. Goodfellow PJ, Billingsley CC, Lankes HA, et al. Combined Microsatellite Instability, MLH1 Methylation Analysis, and Immunohistochemistry for Lynch Syndrome Screening in Endometrial Cancers From GOG210: An NRG Oncology and Gynecologic Oncology Group Study. J Clin Oncol 2015;33:4301-8.

7. Fang WL, Chang SC, Lan YT, et al. Molecular and survival differences between familial and sporadic gastric 
cancers. Biomed Res Int 2013;2013:396272.

8. Ashktorab H, Ahuja S, Kannan L, et al. A meta-analysis of MSI frequency and race in colorectal cancer. Oncotarget 2016;7:34546-57.

9. Nayak SS, Roy P, Arora N, et al. Prevalence estimation of microsatellite instability in colorectal cancers using tissue microarray based methods - A tertiary care center experience. Indian J Pathol Microbiol 2018;61:520-5.

10. Amonkar M, Lorenzi M, Zhang J, et al. Structured literature review (SLR) and meta-analyses of the prevalence of microsatellite instability high (MSI-H) and deficient mismatch repair (dMMR) in gastric, colorectal, and esophageal cancers. J Clin Oncol 2019. doi: 10.1200/ JCO.2019.37.15_suppl.e15074.

11. Zhang Q, Wang L, Ni S, et al. Clinicopathological features and prognostic value of mismatch repair protein deficiency in gastric cancer. Int J Clin Exp Pathol 2018;11:2579-87.

12. Cancer Genome Atlas Research Network. Comprehensive molecular characterization of gastric adenocarcinoma. Nature 2014;513:202-9.

13. Mathiak M, Warneke VS, Behrens HM, et al. Clinicopathologic Characteristics of Microsatellite Instable Gastric Carcinomas Revisited: Urgent Need for Standardization. Appl Immunohistochem Mol Morphol 2017;25:12-24.

14. Karpinska-Kaczmarczyk K, Lewandowska M, Lawniczak M, et al. Expression of Mismatch Repair Proteins in Early and Advanced Gastric Cancer in Poland. Med Sci Monit 2016;22:2886-92.

15. Cho J, Kang SY, Kim KM. MMR protein immunohistochemistry and microsatellite instability in gastric cancers. Pathology 2019;51:110-3.

16. Smyrk TC, Watson P, Kaul K, et al. Tumor-infiltrating lymphocytes are a marker for microsatellite instability in colorectal carcinoma. Cancer 2001;91:2417-22.

17. Buckowitz A, Knaebel HP, Benner A, et al. Microsatellite instability in colorectal cancer is associated with local lymphocyte infiltration and low frequency of distant metastases. Br J Cancer 2005;92:1746-53.

18. Suemori T, Susumu N, Iwata T, et al. Intratumoral CD8+ Lymphocyte Infiltration as a Prognostic Factor and Its Relationship With Cyclooxygenase 2 Expression and Microsatellite Instability in Endometrial Cancer. Int J Gynecol Cancer 2015;25:1165-72.

19. Shen H, Shih J, Hollern DP, et al. Integrated Molecular Characterization of Testicular Germ Cell Tumors. Cell Rep 2018;23:3392-406.

20. Blessin NC, Spriestersbach P, Li W, et al. Prevalence of
CD8(+) cytotoxic lymphocytes in human neoplasms. Cell Oncol (Dordr) 2020;43:421-30.

21. Carcano FM, Lengert AH, Vidal DO, et al. Absence of microsatellite instability and BRAF (V600E) mutation in testicular germ cell tumors. Andrology 2016;4:866-72.

22. Honecker F, Wermann H, Mayer F, et al. Microsatellite instability, mismatch repair deficiency, and BRAF mutation in treatment-resistant germ cell tumors. J Clin Oncol 2009;27:2129-36.

23. Mayer F, Gillis AJ, Dinjens W, et al. Microsatellite instability of germ cell tumors is associated with resistance to systemic treatment. Cancer Res 2002;62:2758-60.

24. Velasco A, Corvalan A, Wistuba II, et al. Mismatch repair expression in testicular cancer predicts recurrence and survival. Int J Cancer 2008;122:1774-7.

25. Velasco A, Riquelme E, Schultz M, et al. Microsatellite instability and loss of heterozygosity have distinct prognostic value for testicular germ cell tumor recurrence. Cancer Biol Ther 2004;3:1152-8; discussion 1159-61.

26. Sommerer F, Hengge UR, Markwarth A, et al. Mutations of BRAF and RAS are rare events in germ cell tumours. Int J Cancer 2005;113:329-35.

27. Necchi A, Bratslavsky G, Corona RJ, et al. Genomic Characterization of Testicular Germ Cell Tumors Relapsing After Chemotherapy. Eur Urol Focus 2020;6:122-30.

28. Rudolph C, Melau C, Nielsen JE, et al. Involvement of the DNA mismatch repair system in cisplatin sensitivity of testicular germ cell tumours. Cell Oncol (Dordr) 2017;40:341-55.

29. Sadigh S, Farahani SJ, Shah A, et al. Differences in PD-L1-Expressing Macrophages and Immune Microenvironment in Testicular Germ Cell Tumors. Am J Clin Pathol 2020;153:387-95.

30. Kononen J, Bubendorf L, Kallioniemi A, et al. Tissue microarrays for high-throughput molecular profiling of tumor specimens. Nat Med 1998;4:844-7.

31. Boland CR, Thibodeau SN, Hamilton SR, et al. A National Cancer Institute Workshop on Microsatellite Instability for cancer detection and familial predisposition: development of international criteria for the determination of microsatellite instability in colorectal cancer. Cancer Res 1998;58:5248-57.

32. Schuster-Bockler B, Lehner B. Chromatin organization is a major influence on regional mutation rates in human cancer cells. Nature 2012;488:504-7.

33. Supek F, Lehner B. Differential DNA mismatch repair underlies mutation rate variation across the human 
genome. Nature 2015;521:81-4.

34. Sesterhenn IA, Davis CJ Jr. Pathology of germ cell tumors of the testis. Cancer Control 2004;11:374-87.

35. Huddart RA, Wooster R, Horwich A, et al. Microsatellite instability in human testicular germ cell tumours. Br J Cancer 1995;72:642-5.

36. Lobo J, Rodrigues A, Guimaraes R, et al. Detailed Characterization of Immune Cell Infiltrate and Expression of Immune Checkpoint Molecules PD-L1/CTLA-4 and MMR Proteins in Testicular Germ Cell Tumors Disclose Novel Disease Biomarkers. Cancers (Basel) 2019;11:1535.

37. Olasz J, Mandoky L, Geczi L, et al. Influence of hMLH1 methylation, mismatch repair deficiency and microsatellite instability on chemoresistance of testicular germ-cell tumors. Anticancer Res 2005;25:4319-24.

38. Lobo J, Pinto C, Pinheiro M, et al. Widening the spectrum of Lynch syndrome: first report of testicular seminoma attributable to MSH2 loss. Histopathology 2020;76:486-9.

39. Staffa L, Echterdiek F, Nelius N, et al. Mismatch repairdeficient crypt foci in Lynch syndrome--molecular alterations and association with clinical parameters. PLoS One 2015;10:e0121980.

40. Yurgelun MB, Goel A, Hornick JL, et al. Microsatellite instability and DNA mismatch repair protein deficiency in
Lynch syndrome colorectal polyps. Cancer Prev Res (Phila) 2012;5:574-82.

41. Fraune C, Simon R, Hoflmayer D, et al. High homogeneity of mismatch repair deficiency in advanced prostate cancer. Virchows Arch 2020;476:745-52.

42. Fraune C, Simon R, Hube-Magg C, et al. MMR deficiency in urothelial carcinoma of the bladder presents with temporal and spatial homogeneity throughout the tumor mass. Urol Oncol 2020;38:488-95.

43. Fraune C, Rosebrock J, Simon R, et al. High homogeneity of MMR deficiency in ovarian cancer. Gynecol Oncol 2020;156:669-75.

44. Cohen R, Hain E, Buhard O, et al. Association of Primary Resistance to Immune Checkpoint Inhibitors in Metastatic Colorectal Cancer With Misdiagnosis of Microsatellite Instability or Mismatch Repair Deficiency Status. JAMA Oncol 2019;5:551-5.

45. Luchini C, Bibeau F, Ligtenberg MJL, et al. ESMO recommendations on microsatellite instability testing for immunotherapy in cancer, and its relationship with PD-1/PD-L1 expression and tumour mutational burden: a systematic review-based approach. Ann Oncol 2019;30:1232-43.
Cite this article as: Dum D, Steurer S, Simon R, Zimmermann PV, Burandt E, Clauditz TS, Fisch M, Rink M, Dahlem R, Höppner W, Zecha H, Doh O, Matthies C, Wilczak W, Sauter G, Fraune C. Mismatch repair deficiency occurs very rarely in seminomas. Transl Androl Urol 2021;10(3):1048-1055. doi: $10.21037 /$ tau-20-1355 